\section{mTOR, la cible fonctionnelle de la rapamycine}

\author{
Louis-André Julien, Philippe P. Roux
}

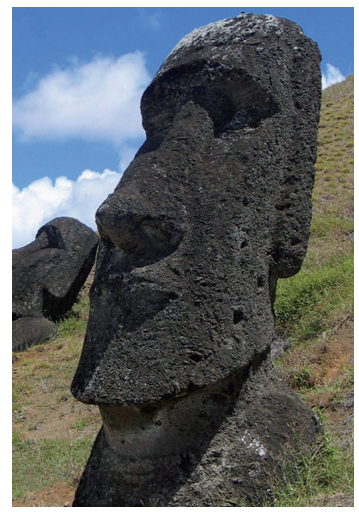

Institut de recherche en immunologie et en cancérologie (IRIC), Département de pathologie et biologie cellulaire, Faculté de médecine, Université de Montréal, CP 6128, Succursale Centre-ville, Montréal (Québec), H3C 3J7 Canada. philippe.roux@umontreal.ca

identifièrent plusieurs levures mutantes de la levure Saccharomyces cerevisiae résistantes à la rapamycine; la plupart des mutations étaient situées dans le gène FPRI, l'orthologue de FKBP12, mais deux d'entre elles ciblaient de nouveaux gènes, qui furent nommés TORI et TOR2, pour target of rapamycin [3]. En 1994, plusieurs groupes isolèrent, à partir de cellules humaines, une protéine qui s'associait au complexe FKBP12-rapamycine, qui fut nommée FRAP (FKBP rapamycinassociated protein) et RAFT (rapamycin and FKBP12 target), et dont la séquence était similaire à celle du produit des gènes TORI et TOR2 identifiés chez la levure $[4,5]$. Bien qu'elle soit ciblée par un complexe FKBP12-rapamycine, la protéine FRAP/RAFT chez les mammifères est couramment appelée mTOR pour mammalian TOR. Dans cet article de synthèse, nous décrirons brièvement les mécanismes de régulation et les fonctions cellulaires de mTOR.

\section{mTOR, la cible pharmacologique du complexe rapamycine-FKBP12}

La délétion homozygote du gène FRAP, situé sur le chromosome lp36.2 chez l'humain, est létale très tôt au cours du développement embryonnaire murin $(\varepsilon 5,5)$. L'ARN messager (ARNm) codant pour mTOR est exprimé de façon ubiquitaire dans la plupart des tissus et types cellulaires. Le produit du gène FRAP est une sérine/thréonine kinase atypique de la famille des phosphatidylinositol-3-kinase (PI3K)-related kinase (PIKK) [6]. En plus du domaine kinase, mTOR contient une vingtaine de répétitions HEAT (Huntingtin, elongation factor 3, PR65/A subunit of protein phosphatase 2A, TOR). Leur fonction demeure incomprise mais pourrait régir des interactions protéine-protéine. Pour sa part, mTOR contient aussi des domaines FAT (FRAP/ATM/TRRAP) et FATC (FAT carboxy-terminal) communs à tous les membres de la famille PIKK et importants pour la phosphorylation 


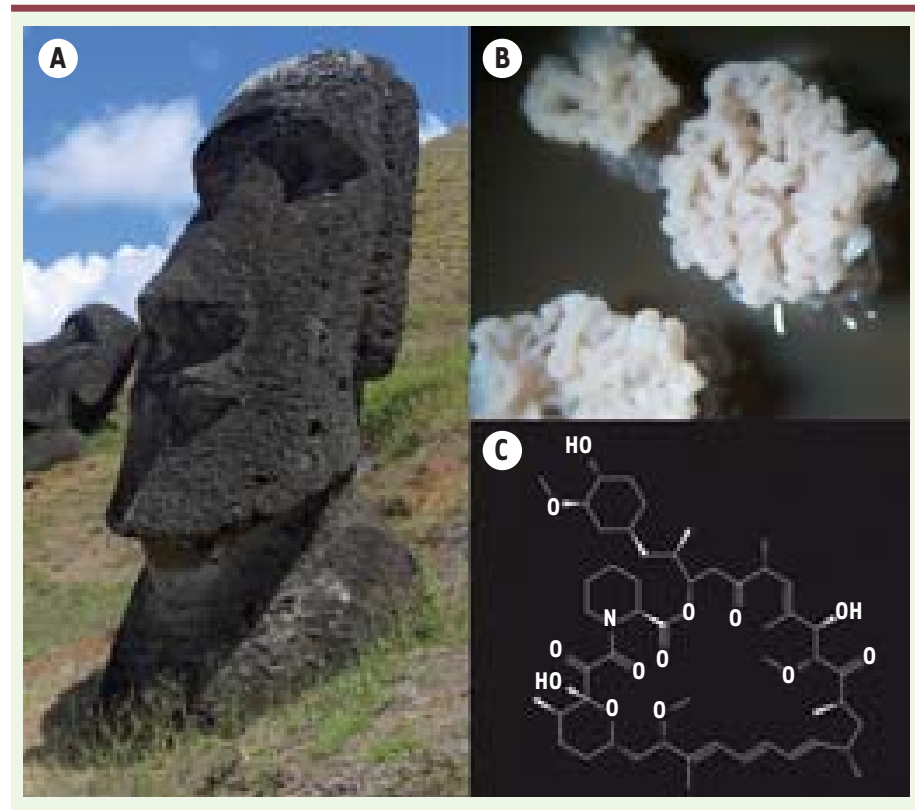

de substrats par mTOR via un mécanisme inconnu [6]. De plus, mTOR possède un domaine FRB (FKBP12-rapamycin binding) qui est la cible du complexe FKBP12-rapamycine, mais les mécanismes par lesquels cette interaction inhibe l'activité de mTOR demeurent incompris. Enfin, une forme fonctionnelle de mTOR (mTOR $\beta$ ) provenant d'un épissage alternatif a récemment été identifiée et pourrait agir en tant que proto-oncogène [7].

\section{mTOR forme deux complexes intracellulaires}

La protéine kinase mTOR est le centre catalytique de deux complexes multiprotéiques fonctionnellement distincts, conservés chez tous les eucaryotes et nommés mTORCl et mTORC2 [8, 9] (Figure 2). Lorsqu'elle est associée à Raptor (regulatory associated protein of TOR) et à mLST8 (mammalian lethal with secl3 protein 8), mTOR forme le complexe mTORC1. Ce complexe interagit avec Deptor (DEP domaincontaining mTOR-interacting protein), FKBP38 et PRAS40 (prolinerich Akt substrate of $40 \mathrm{kDa}$ ), régulateurs négatifs de mTORCl. Pour former mTORC2, mTOR interagit avec les protéines Rictor (rapamycininsensitive companion of TOR), $\operatorname{Sin} 1$ (stress-activated map kinaseinteracting protein 1 ) et $\mathrm{mLST}$ 8. De plus, mTORC2 s'associe aussi avec Deptor, qui réprime son activité, ainsi qu'avec PPR5/Protor, dont la fonction demeure inconnue. Lorsqu'elle est liée à FKBP12, la rapamycine inhibe spécifiquement mTORCl, mais la cause exacte de cette spécificité demeure encore incomprise.

\section{mTORCl et la synthèse protéique}

\section{Régulation de l'activité de mTORCl}

L'activité de mTORCl est modulée par la disponibilité en nutriments, l'état énergétique de la cellule et par les facteurs de croissance (mitogènes et hormones) [10] (Figure 2). De plus, régulée par plusieurs
Figure 1. De Rapa Nui à la rapamycine. A. Un moaï, statue gigantesque que l'on trouve par centaines sur l'île de Pâques (photographie d'Artemio Urbina). B. La rapamycine est un produit de la bactérie Streptomyces hygroscopicus qui fut isolée à partir d'un échantillon de sol de l'île de Pâques. Photographie d'une souche de bactérie Streptomyces prise par le Dr Ramón Santamaría, IMB/CSIC-USAL, Espagne. C. Structure chimique de la rapamycine $\left(\mathrm{C}_{51} \mathrm{H}_{79} \mathrm{NO}_{13}\right)$.

proto-oncogènes et suppresseurs de tumeurs, mTORCl est activée dans un nombre croissant de néoplasmes. Liée au GTP, la petite GTPase Rheb (Ras homologue enriched in brain) interagit avec mTOR et active spécifiquement la signalisation mTORC1 [11]. Le complexe dimérique formé de TSCl (hamartine) et TSC2 (tubérine) est un régulateur négatif important de $\mathrm{mTORCl}$ puisque son activité GAP (GTPase activating protein) hydrolyse la molécule de GTP liée à Rheb activée.

Le complexe TSCl/2 constitue un carrefour ciblé par plusieurs voies de signalisation. L'activation de PI3K par les facteurs de croissance mène à l'accumulation dans la membrane plasmique de phosphatidylinositol-3,4,5triphosphate $\left(\mathrm{PIP}_{3}\right)$, phospholipide important pour le recrutement et l'activation de PDKl (phosphoinositidedependent kinase-1) et de l'oncogène Akt [11]. Une fois activé, Akt phosphoryle TSC2 et inhibe son activité GAP, permettant l'activation de mTORCl par Rheb. Akt active également mTORCl en phosphorylant PRAS40. De plus, les kinases ERK (extracellular signal-regulated kinase) et RSK ( 990 ribosomal S6 kinase), en aval de la voie de signalisation de la petite GTPase Ras, activent mTORCl en phosphorylant et inactivant TSC2 [12]. Enfin, les kinases RSK augmentent l'activité de mTORCl en phosphorylant directement Raptor [13].

Lorsque les niveaux énergétiques de la cellule sont faibles, I'AMPK (AMP-activated protein kinase) diminue l'activité de mTORCl en phosphorylant TSC2 et Raptor. De son côté, mTORCl est également sensible à la disponibilité en nutriments, notamment en acides aminés, qui l'activent indépendamment du complexe TSCl/2. En effet, les acides aminés envoient mTORCl à la surface d'un compartiment membranaire intracellulaire qui contient Rheb. Ce mécanisme, qui requiert les petites GTPases Rag, est nécessaire pour l'activation de mTORCl par Rheb [14]. De plus, les acides aminés activent mTORCl grâce à une PI3K de classe III, hVps34 (human vacuolar protein sorting 34), activée par la voie $\mathrm{Ca}^{2+} /$ calmoduline [15]. En outre, I'hypoxie diminue l'activité de mTORCl via un mécanisme requérant REDDl (regulated in development and DNA damage 
response gene 1) et REDD2 [16]. L'inhibition de $\mathrm{mTORCl}$ en réponse à l'hypoxie, à l'absence de nutriments ou à de faibles niveaux énergétiques, est dominante sur les signaux qui l'activent, empêchant son activation lorsque les conditions ne sont pas adéquates pour la croissance cellulaire. Enfin, l'acide phosphatidique (PA), produit lipidique de la phospholipase-D (PLD), lie le domaine FRB de mTOR et semble être un régulateur important de mTORCl [17].

\section{La traduction des ARNm}

La traduction des ARNm est une étape déterminante de l'expression génique dont dépendent la différenciation, la croissance et la prolifération cellulaires. II faut souligner que mTORCl est un régulateur majeur de la synthèse protéique puisqu'il régit la formation du complexe d'initiation de la traduction elF4F (elongation initiation factor $4 F)$ [10], formé des sous-unités elF4E, elF4A et elF4G. La sous-unité elF4E est un oncogène surexprimé dans plusieurs types de néoplasmes car il promeut la traduction des ARNm en liant directement la structure de la coiffe. La formation de elF4F à la coiffe est inhibée par les protéines $4 \varepsilon$-BP ( $4 \varepsilon$-binding proteins), qui déplacent elF4G pour se lier à elF4E. En phosphorylant les $4 \varepsilon-B P$, mTORCl inhibe leur liaison à elF4E et favorise la formation du complexe elF4F.

Le complexe mTORCl régule un autre effecteur de la croissance cellulaire en phosphorylant le motif hydrophobique de S6K (p70 ribosomal S6 kinase), une kinase de la famille AGC (protéine kinase $A, G$ et $C$ ). Une fois activée, S6K phosphoryle elF4B, favorisant son association au complexe de pré-initiation de la traduction [10], et d'autres substrats qui régissent la synthèse protéique, dont la protéine ribosomale $S 6$ de la sous-unité $40 S$ du ribosome, eEF2K (eukaryotic translation elongation factor 2 kinase) et PDCD4 (programmed cell death protein 4).

\section{La biogenèse des ribosomes}

mTORCl coordonne la biosynthèse des ribosomes en entraînant la traduction d'ARNm possédant une séquence terminale en 5' de 6 à 12 pyrimidines, nommés 5'TOP (5’ terminal oligo-pyrimidine) [10]. Cette classe d'ARNm code pour des facteurs d'élongation de la traduction, certains facteurs d'initiation ainsi que la majorité des protéines ribosomales. La façon dont mTORCl conduit la traduction de ces ARNm reste inconnue. De plus, mTORCl module l'activité de I'ARN polymérase I, qui transcrit les $A R N r$, en favorisant l'activité du régulateur transcriptionnel TIF-1A (transcriptional intermediary factor lA). Même si les mécanismes ne sont pas connus, il semble que mTORCl module la synthèse de l'ARN $5 S$ et des ARN de transfert (ARNt) par l'ARN polymérase III. Enfin, chez la levure, l'homologue de mTORCl (TORI) lie le promoteur du gène de l'ARNr 35S, ce qui est important pour sa synthèse et pour la croissance cellulaire [18].

\section{Autres fonctions importantes de mTORC1}

Le complexe mTORCl participe à la régulation de l'autophagie, mécanisme de dégradation non spécifique provoqué par une carence en nutriments et qui permet à la cellule de recycler de vieux organites et protéines. Chez la levure, TORl bloque l'association entre ATG13 (autophagic specific gene 13) et ATGl [19], processus essentiel à la formation d'autophagosomes, tandis que la rapamycine déclenche l'autophagie dans des tissus murins et humains, indiquant que mTORCl est un inhibiteur de l'autophagie [20]. L'hypothèse selon laquelle l'autophagie pourrait permettre à la cellule cancéreuse de survivre et de contourner l'apoptose en absence de nutriments demeure sujette à débat et le rôle entre l'autophagie et mTOR dans la tumorigenèse reste à déterminer.

Le complexe mTORCl module l'expression de plusieurs transporteurs de nutriments de façon transcriptionnelle et post-traductionnelle, dont celle du transporteur de glucose GLUTl (glucose transporter 1), de plusieurs transporteurs d'acides aminés et du récepteur de la transferrine [21]. Le transport du glucose et de nutriments étant un processus souvent accéléré dans les cellules cancéreuses, ces fonctions de mTORCl pourraient jouer un rôle dans la tumorigenèse.

\section{Fonctions et régulation de mTORC2}

\section{Régulation de l'oncogène Akt}

L'une des preuves de l'importance du rôle que joue mTOR dans le cancer est attestée par la découverte que l'oncogène Akt, régulateur majeur de la prolifération et de la survie cellulaires, est directement phosphorylé et activé par mTORC2 [22] (Figure 2). En effet, l'activation d'Akt par les facteurs de croissance nécessite la phosphorylation d'une thréonine dans la boucle d'activation (Thr308) par PDKl et d'une sérine située dans un site hydrophobe (Ser473) par mTORC2 [22]. D'ailleurs, mTORC2 phosphoryle un site similaire sur les kinases SGKl et PKC $\alpha$, ce qui signifie qu'une des fonctions de mTOR consiste à activer plusieurs kinases de la famille AGC [23]. De plus, des études récentes indiquent que mTORC2 pourrait jouer un rôle dans la phosphorylation du turn motif de plusieurs kinases AGC lors de leur synthèse de novo; ce phénomène serait important pour leur stabilité [23]. Enfin, mTORC2 joue un rôle dans la structuration du cytosquelette d'actine puisque la diminution de son expression empêche la polymérisation de l'actine et l'expansion cellulaire [9].

\section{Régulation de l'activité de mTORC2}

Les mécanismes qui modulent l'activité de mTORC2 sont peu connus. Ce complexe semble être sous la domination de PI3K, car les facteurs de croissance qui l'activent provoquent la phosphorylation d'Akt dépendante de mTORC2. Plusieurs mécanismes de rétrocontrôle déclenchés par mTORCl semblent inhiber l'activité de mTORC2 [11]. Ainsi, bien que Rheb n'interagisse pas 


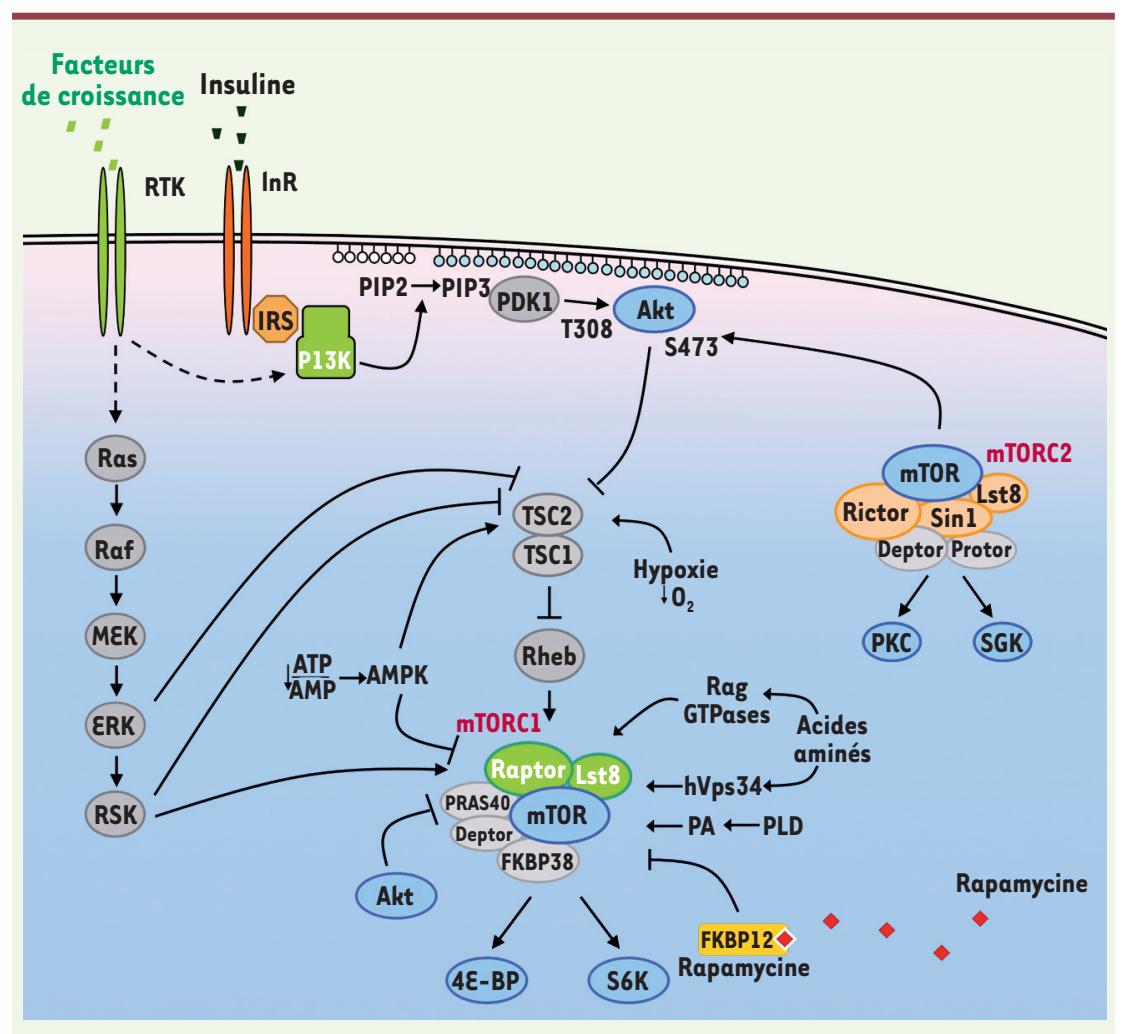

Figure 2. Représentation schématique de la voie mTOR. La sérine/thréonine mTOR fait partie de deux complexes fonctionnellement distincts définis par leurs sous-unités spécifiques, Raptor (mTORC1) et Rictor (mTORC2). Les facteurs de croissance qui activent les récepteurs à activité tyrosine kinase (RTK) et le récepteur de l'insuline $(\ln R)$ activent PI3K, ce qui entraîne la formation de PIP3 dans la membrane plasmique et l'activation de mTORC2 et d'Akt. mTORC2 régule également PKC et SGK. Une fois activé, Akt phosphoryle TSC2 et PRAS40, ce qui induit l'activation de Rheb et de mTORCl. mTORCl, la cible du complexe FKBP12-rapamycine, régule la synthèse protéique en phosphorylant $4 \varepsilon-B P$ et S6K. L'activation de la voie Ras/MAPK mène également à l'activation de $\mathrm{mTORCl}$ après la phosphorylation de TSC2 par ERK et RSK et celle de Raptor par RSK. L'acide phosphatidique (PA) semble être un régulateur important de mTORCl. Enfin, l'absence d'acides aminés, l'hypoxie et de faibles niveaux d'ATP inhibent l'activation de mTORCl.

avec mTORC2, il semble qu'il soit un antagoniste de son activité : il s'agit probablement d'une conséquence de l'induction de mécanismes de rétro-inhibition lancés par mTORCl. Toutefois, le complexe TSCl/2 semble être un activateur de mTORC2 indépendamment des mécanismes de rétrocontrôle amorcés par mTORCl car il se lie directement à mTORC2 et l'active par un mécanisme encore méconnu [24].

L'activation de mTORCl et de S6K joue un rôle important dans la mise en œuvre de mécanismes de rétrocontrôle qui diminuent l'activation de PI3K et d'Akt [11]. La phosphorylation d'Akt sur la Ser473 est inhibée et insensible à l'insuline dans des cellules embryonnaires de souris $\mathrm{TSC2}^{-/-}$dans lesquelles l'activité mTORCl et S6K est constitutivement élevée. Un traitement par la rapamycine rétablit la sensibilité de ces cellules à l'insuline et permet la phosphorylation d'Akt, indiquant que les mécanismes de rétro-inhibition sont provoqués par mTORCl. En effet, la phosphorylation des protéines adaptatrices IRS (insulin receptor substrate 1) par mTORCl et S6K a pour effet de diminuer leur stabilité et d'empêcher le recrutement de PI3K [25]. De plus, des études plus récentes ont démontré que Rictor est phosphorylé par S6K1, ce qui inhibe l'activité de mTORC2 et diminue la phosphorylation et l'activation d'Akt [26-28].

\section{Nouvelle génération d'inhibiteurs de mTOR}

La délétion du gène FRAP semble avoir un effet plus marqué que la rapamycine sur la croissance et la prolifération cellulaires, suggérant que certaines fonctions de mTOR ne sont pas entièrement inhibées par la rapamycine [29]. La deuxième génération d'inhibiteurs mTOR est constituée d'antagonistes de I'ATP, dont la Torinl et le PP242 [9], qui inhibent mTOR indépendamment du fait qu'il fasse partie de mTORCl ou mTORC2. De plus, ces inhibiteurs du domaine catalytique de mTOR ont permis d'identifier une activité $\mathrm{mTORCl}$ résistante à la rapamycine; d'ailleurs, ils bloquent plus fortement la croissance et la prolifération cellulaire que la rapamycine à elle seule. Somme toute, ces inhibiteurs seront utiles pour déterminer les rôles biologiques exacts de mTOR et se profilent comme des agents thérapeutiques prometteurs pour le traitement du cancer.

\section{Conclusions et perspectives}

Le rôle qu'exerce mTOR dans plusieurs pathologies justifie la mise au point d'agents thérapeutiques ciblant son activité. Certes la rapamycine et ses analogues représentent des agents dotés d'un fort potentiel pour le traitement de plusieurs cancers, cependant ces molécules semblent inefficaces lorsqu'elles sont utilisées seules, probablement parce qu'elles ne ciblent que mTORCl. La deuxième génération d'inhibiteurs de mTOR, constituée d'antagonistes de I'ATP de son domaine kinase, ciblent mTORCl et mTORC2: ils inhibent ainsi plus efficacement les fonctions cellulaires de mTOR. Dès lors, ces nouvelles molécules pourraient être plus efficaces pour le traitement de certains cancers. 
La rapamycine a été découverte il y a une quarantaine d'années; nos connaissances sur la régulation et les fonctions de mTOR sont plus récentes et demeurent limitées. Le défi des prochaines années consistera à mieux caractériser cette voie de signalisation afin d'améliorer les stratégies contre le cancer fondées sur l'inhibition de mTOR. $\diamond$

\section{SUMMARY}

mTOR, the mammalian target of rapamycin

The discovery of rapamycin from a soil sample on Easter Island in the mid 60's marked the beginning of an exciting field of research in cell biology and medicine. While it was first used as an antifungal and as an immunosuppressive drug, more recent studies confirmed rapamycin's antiproliferative properties over a variety of solid tumors. Research aimed at identifying its mechanism of action uncovered mTOR (mammalian target of rapamycin), a protein kinase that regulates mRNA translation and protein synthesis, an essential step in cell division and proliferation. Recent evidence suggests a more complex role for mTOR in the regulation of several growth factor-stimulated protein kinases, including the proto-oncogene Akt. This article reviews mTOR function and regulation, and briefly details the future challenges for anticancer therapies based on mTOR inhibition. $\diamond$

\section{CONFLIT D'INTÉRÊTS}

Les auteurs déclarent n'avoir aucun conflit d'intérêts concernant les données publiées dans cet article.

\section{RéFÉRENCES}

1. Vezina C, Kudelski A, Sehgal SN. Rapamycin (AY-22,989), a new antifungal antibiotic. I. Taxonomy of the producing streptomycete and isolation of the active principle. J Antibiot (Tokyo) 1975; $28: 721-6$.

2. Harding MW, Galat A, Uehling DE, Schreiber SL. A receptor for the immunosuppressant FK506 is a cis-trans peptidyl-prolyl isomerase. Nature $1989 ; 341: 758-60$.

3. Heitman J, Movva NR, Hall MN. Targets for cell cycle arrest by the immunosuppressant rapamycin in yeast. Science $1991 ; 253: 905-9$.

4. Sabatini DM, Erdjument-Bromage $H$, Lui M, et al. RAFTl: a mammalian protein that binds to FKBP12 in a rapamycin-dependent fashion and is homologous to yeast TORs. Cell 1994 ; $78: 35-43$.

5. Brown $\varepsilon$ J, Albers MW, Shin TB, et al. A mammalian protein targeted by Gl-arresting rapamycinreceptor complex. Nature $1994 ; 369: 756-8$

6. Lempiainen $\mathrm{H}$, Halazonetis TD. Emerging common themes in regulation of PIKKs and PI3Ks. EMBOJ $2009 ; 28: 3067-73$.

7. Panasyuk G, Nemazanyy I, Zhyvoloup A, et al. mTORbeta splicing isoform promotes cell proliferation and tumorigenesis. J Biol Chem 2009; 284 : 30807-14.

8. Dunlop $\varepsilon A$, Tee AR. Mammalian target of rapamycin complex 1 : signalling inputs, substrates and feedback mechanisms. Cell Signal $2009 ; 21: 827-35$.
9. Guertin DA, Sabatini DM. The pharmacology of mTOR inhibition. Sci Signal $2009 ; 2$ : pe24.

10. Ma XM, Blenis J. Molecular mechanisms of mTOR-mediated translational control. Nat Rev Mol Cell Biol $2009 ; 10$ : 307-18.

11. Huang J, Manning BD. A complex interplay between Akt, TSC2 and the two mTOR complexes. Biochem Soc Trans $2009 ; 37: 217-22$.

12. Carriere A, Ray H, Blenis J, Roux PP. The RSK factors of activating the Ras/ MAPK signaling cascade. Front Biosci $2008 ; 13: 4258-75$.

13. Carrière A, Cargnello M, Julien LA, et al. Oncogenic MAPK signaling stimulates mTORCl activity by promoting RSK-mediated raptor phosphorylation. Curr Biol 2008; 18 : 1269-77.

14. Kim $\varepsilon$, Guan KL. RAG GTPases in nutrient-mediated TOR signaling pathway. Cell Cycle $2009 ; 8$ : 1014-8.

15. Gulati P, Gaspers LD, Dann SG, et al. Amino acids activate mTOR complex 1 via Ca2+/CaM signaling to hVps34. Cell Metab 2008; 7: 456-65.

16. Brugarolas J, Lei K, Hurley RL, et al. Regulation of mTOR function in response to hypoxia by REDDI and the TSC1/TSC2 tumor suppressor complex. Genes Dev $2004 ; 18: 2893-904$.

17. Fang $Y$, Vilella-Bach M, Bachmann R, et al. Phosphatidic acid-mediated mitogenic activation of mTOR signaling. Science $2001 ; 294$ : 1942-5.

18. Li H, Tsang CK, Watkins M, et al. Nutrient regulates Torl nuclear localization and association with rDNA promoter. Nature 2006 ; 442 : 1058-61.

19. Kamada Y, Funakoshi T, Shintani T, et al. Tor-mediated induction of autophagy via an Apgl protein kinase complex.J Cell Biol 2000; $150: 1507-13$.

20. Paglin S, Lee NY, Nakar C, et al. Rapamycin-sensitive pathway regulates mitochondrial membrane potential, autophagy, and survival in irradiated MCF-7 cells. Cancer Res $2005 ; 65: 11061-70$.

21. Edinger AL. Controlling cell growth and survival through regulated nutrient transporter expression. Biochem/ $2007 ; 406$ : 1-12.

22. Bhaskar PT, Hay N. The two TORCs and Akt. Dev Cell $2007 ; 12$ : 487-502.

23. Alessi DR, Pearce LR, Garcia-Martinez JM. New insights into mTOR signaling: mTORC2 and beyond. Sci Signal 2009; 2 : pe27.

24. Huang J, Dibble CC, Matsuzaki M, Manning BD. The TSC1-TSC2 complex is required for proper activation of mTOR complex 2. Mol Cell Biol 2008 ; 28: 4104-15.

25. Zick Y. Ser/Thr phosphorylation of IRS proteins: a molecular basis for insulin resistance. Sci STKE 2005; 2005 : pe4.

26. Dibble CC, Asara JM, Manning BD. Characterization of Rictor phosphorylation sites reveals direct regulation of mTOR complex 2 by S6K1. Mol Cell Biol $2009 ; 29: 5657-70$.

27. Julien LA, Carrière A, Moreau J, Roux PP. mTORCl-activated S6K1 phosphorylates Rictor on threonine 1135 and regulates mTORC2 signaling. Mol Cell Biol 2010 ; 30 : 908-21.

28. Treins C, Warne PH, Magnuson MA, et al. Rictor is a novel target of p70 S6 kinase-1. Oncogene $2010 ; 29: 1003-16$.

29. Murakami M, Ichisaka T, Maeda M, et al. mTOR is essential for growth and proliferation in early mouse embryos and embryonic stem cells. Mol Cell Biol $2004 ; 24: 6710-8$.

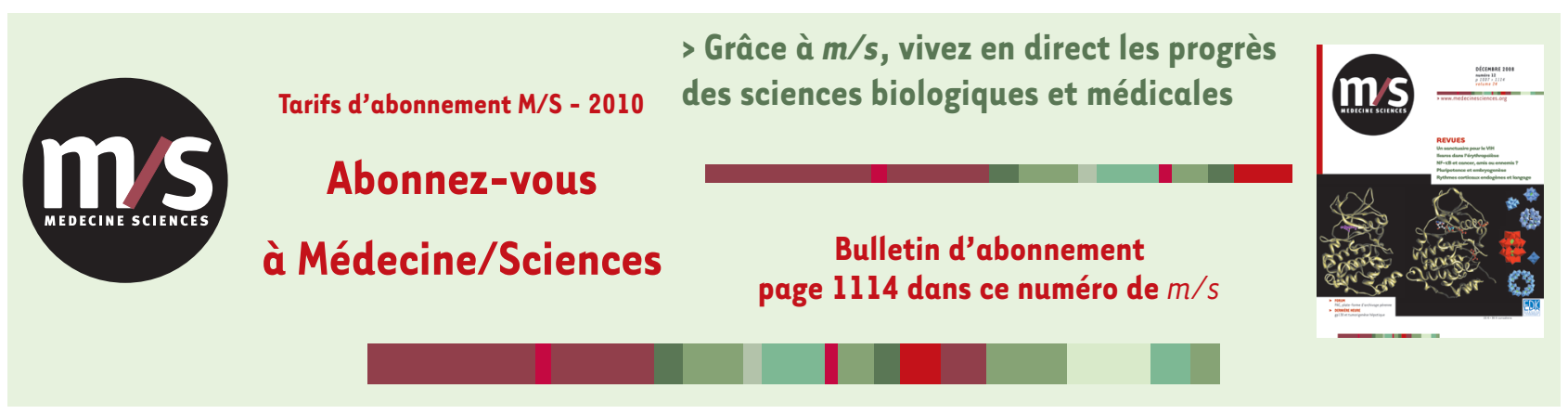

TIRÉS À PART

P.P. Roux 